\title{
Spiking Neural Network-Based Radar Gesture Recognition System Using Raw ADC Data
}

This paper was downloaded from TechRxiv (https://www.techrxiv.org).

LICENSE

CC BY 4.0

SUBMISSION DATE / POSTED DATE

23-02-2022 / 01-03-2022

CITATION

Arsalan, Muhammad; Santra, Avik; Issakov, Vadim (2022): Spiking Neural Network-Based Radar Gesture Recognition System Using Raw ADC Data. TechRxiv. Preprint. https://doi.org/10.36227/techrxiv.19221513.v1

$\mathrm{DOI}$

10.36227/techrxiv.19221513.v1 


\title{
Spiking Neural Network-Based Radar Gesture Recognition System Using Raw ADC Data
}

\author{
Muhammad Arsalan ${ }^{12 *}$, Avik Santra ${ }^{2 * *}$, and Vadim Issakov ${ }^{12 *}$ \\ ${ }^{1}$ Infineon Technologies AG, Neubiberg, 85579, Germany \\ ${ }^{2}$ Fakultät für Elektrotechnik, Informationstechnik, Physik, Technische Universität Braunschweig, Braunschweig, 38106, Germany \\ * Student Member, IEEE \\ ** Senior Member, IEEE
}

\begin{abstract}
One of the main challenges in developing embedded radar-based gesture recognition systems is the requirement of energy efficiency. To facilitate this, we present an embedded gesture recognition system using a $60 \mathrm{GHz}$ frequency modulated continuous wave radar using spiking neural networks (SNNs) applied directly to raw ADC data. The SNNs are sparse in time and space, and event-driven which makes them energy-efficient. In contrast to the previous state-of-the-art methods, the proposed system is only based on the raw ADC data of the target thus avoiding the overhead of performing the slow-time and fast-time Fourier transforms (FFTs). Furthermore, the pre-processing slow-time FFT is mimicked in the proposed SNN architecture, where the proposed model processing speed of $\mathbf{1 2} \mathbf{~ m s}$ advances the state-of-the-art by a factor of 17.7. The experimental results demonstrate that despite the simplification the proposed implementation achieves recognition accuracy of $\mathbf{9 8 . 1} \%$, which is comparable to the conventional approaches.
\end{abstract}

Index Terms-Gesture sensing, Spiking neural networks, Human computer interface, Nengo

\section{INTRODUCTION}

Hand gesture sensing (HGS) has been an active research field over the last two decades because of its contactless nature and easy interfacing with machines [1] as compared to the click and touch devices. HGS contributes to many applications such as TVs, smart home devices, automotive applications, and virtual reality. With the advancement in camera-based recognition systems, HGS is primarily dominated by camera-based systems [2], [3]. However, user privacy concerns and the requirement of environmental conditions (illumination, weather etc.) limits the use of camera-based systems. Contrastingly, non-vision-based systems provide a cumbersome user experience due to their wearable nature.

Recently, contactless non-vision-based systems, such as radarbased systems, garnered a lot of attention because of their insensitive nature to the illumination conditions, invariance to hand occlusions, simpler signal processing pipeline, privacy-preserving features, ability to work within an enclosure, and their sensitivity to fine-grained gestures. There are two fundamental research directions to radarbased gesture systems: 1) building efficient miniature hardware for generating high-fidelity target data [4]-[6], and 2) the signal processing pipeline driven by deep learning to extract meaningful information from the target data of the user's intent [7]-[13]. Although the conventional deep neural networks (deepNets) approaches are quite promising in terms of gesture detection and recognition, energy consumption is still an issue making them unfavorable for portable devices. The majority of the energy in deepNets solutions is consumed by the multiply-accumulate (MAC) operations between layers [14]. Thus researchers focus primarily on the reduction of MACs by using smaller networks, using pruning techniques and quantizing the weights [15], [16].

Contrary to traditional deepNets solutions, we propose a gesture system based on spiking neural networks ( $\mathrm{SNNs}$ ), where the information is encoded by spike timing, including latencies and spike rates. Furthermore, information is only transmitted when the neuron potential reaches a specific threshold, hence making the transmission sparse in nature with 1-bit activity, which substantially reduces the amount of data communicated between neurons. Moreover, the node accumulates information only when it receives a spike and the multipliers in MAC arrays are replaced with adders making the SNNs energy-efficient. However, SNNs are hard to train due to the non-differentiable transfer function hindering backpropagation, thus requiring a suitable learning mechanism for training the SNNs. There exists different spiking neuron models, which are defined at different levels of abstraction, but the leaky integrate-and-file (LIF) model is the most popular because of its biological plausibility and simple implementation requiring fewer computations. (floating-point operations).

The main contributions of this letter are as follows:

- We propose an end-to-end pipeline for a radar-based gesture sensing system from raw data using SNN.

- Compared to previous approaches [17]-[19] which work on Doppler images, the propose approach is applied to the raw ADC data, thus avoiding the overhead of performing slow-time and fast-time FFTs.

- We proposed a novel SNN architecture where the signal preprocessing (slow-time FFT) is mimicked in SNN.

- The overall end-to-end latency is substantially reduced (by a factor of 17.7) compared to previous approaches [17], [18].

\section{PROPOSED RECOGNITION SYSTEM}

\section{A. Hardware}

The FMCW radar chipset BGT60TR13C [20] by Infineon Technologies has been used in this work. The radar chipset and its simplified block diagram are shown in Fig. 2(a) and (b) respectively. The chipset embodies transmitters 1 transmit Tx path antenna and 3 receive path antennas, mixer, and Analog-to-Digital Converter (ADC). It as an external phase-locked loop that controls the linear frequency sweep. A $80 \mathrm{MHz}$ reference oscillator controls the loop with frequency divider output pin and the Finite State Machine (FSM) is clocked by a reference clock running at $80 \mathrm{MHz}$ [21]. The voltage-controlled oscillator (VCO) is enabled by varying the tune voltage $V_{\text {tuned }}$ from 1 to $4.5 \mathrm{~V}$ to generate a linear frequency sweep from $57 \mathrm{GHz}$ to $63 \mathrm{GHz}$. For memory readout Serial Peripheral Interface (SPI) and Queued Serial Peripheral Interface (QSPI) is embodied in the chipset allowing a maximum data transfer upto $200 \mathrm{Mb} / \mathrm{s}(4 \times 50 \mathrm{Mb} / \mathrm{s})$. 


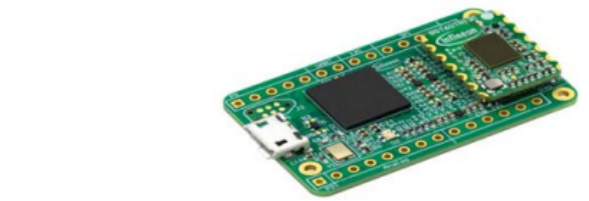

(a)

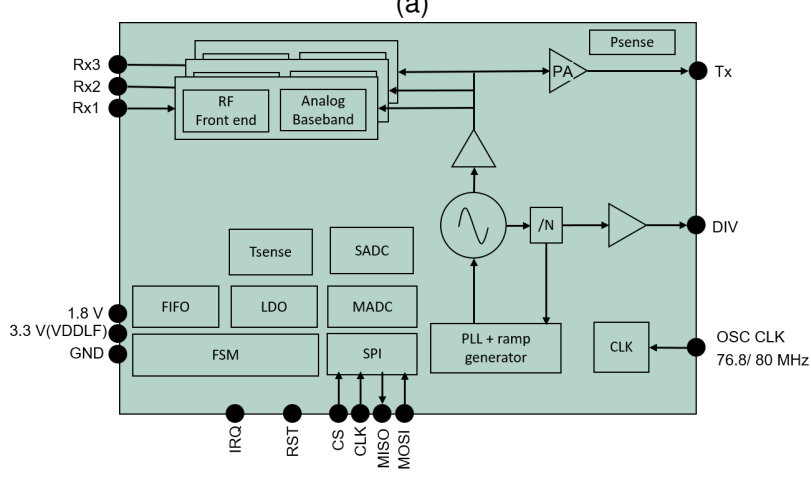

(b)

Fig. 1: a) Infineon's BGT60TR13C FMCW radar chipset. b) Simplified block diagram of Infineon's BGT60TR13C radar chipset.

For streaming out of the data, the FSM generate an interrupt (IRQ) flag when the thresold set by host is met in the memory. The chipset is capable of transmitting a signal up to $6 \mathrm{GHz}$ bandwidth where the transmitted signal and received signals are time domain multiplied and fed forward for further processing. In our experiment we have used only single Rx channel.

\section{B. Signal processing}

1) Moving target indication filtering: The raw ADC data is collected across a chirp (fast-time) and arranged in rows along with the frame (slow-time). In the FMCW radar, the reflection from stationary objects in the surroundings can subdue the hand reflection. Therefore, we apply moving target indication (MTI) to suppress the reflection from these stationary objects and leakage. At each frame $j$, a moving average filter is applied to the fast-time $F(j)$, given mathematically as:

$$
\begin{array}{r}
F(j)=F(j)-S(j-1) \\
S(j)=\alpha \cdot F(j)+(1-\alpha) \cdot S(j-1)
\end{array}
$$

where $\alpha$ denotes forget factor set to 0.01 . This filtered fast-time data is fed to the target detection block.

2) Target detection: Thresholding is used to perform target detection and selection on the filtered fast-time data. The threshold is determined by using the mean value of the fast-time data with a scaling factor. For example, the threshold $\Gamma$ at frame $j$ is given by:

$$
\Gamma_{j}=\beta \times \sum_{n=1}^{N_{b}} F_{n}(j),
$$

where $\beta$ is the scaling factor set to 3 , chosen empirically for the trade-off between false positive and probability of detection. $n$ is index along range bins and $N_{b}$ is the number of range bins. Once a moving target is detected, the filtered raw ADC data is accumulated and fed into SNN for gesture recognition.

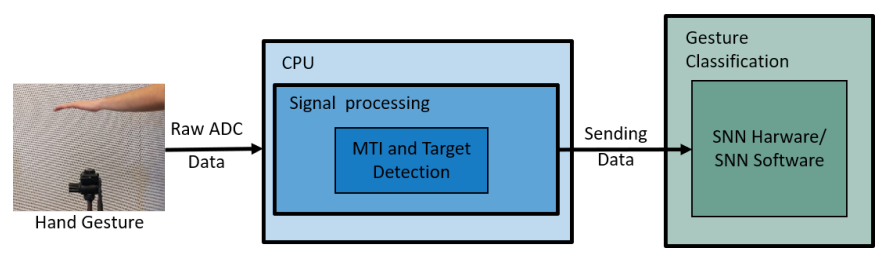

Fig. 2: Proposed signal processing chain.

\section{System Design}

Figure 2(b) shows the experimental setup for recording hand gestures. A gesture is made in front of a $60 \mathrm{GHz}$ radar chipset configured with the system (and derived) parameters shown in Table 1.

Table 1: System Parameters Summary

\begin{tabular}{lll}
\hline Parameter & Symbol & Value \\
\hline Number of ADC samples & $N T S$ & 64 \\
Number of Chirps & $N_{c}$ & 1 \\
Chirp Time & $T_{c}$ & $32 \mu \mathrm{s}$ \\
Number of Transmit Antennas & $N_{T X}$ & 1 \\
Number of Receive Antennas & $N_{R X}$ & 1 \\
Total Bandwidth & $B$ & $5 \mathrm{GHz}$ \\
Frame Time & $T_{f}$ & $75.476 \mathrm{~ms}$ \\
Azimuth Antenna Field of View & $\Theta_{F O V}$ & $70^{\circ}$ \\
\hline
\end{tabular}

The radar chipset is connected to a PC through a USB device to store the ADC data. Gesture recording is automated and starts as soon as the hand enters the field of view of the radar and the frame count starts. Each gesture is recorded and labeled for 32 consecutive frames with a large inter-class variance. Our dataset contains some gestures of high variance, and gestures with shorter number frames (less than 32) are appended with zeros chirp values.

\section{SPIKING NEURAL NETWORKS}

Although inspired by the biological nervous systems, Artificial neural networks (ANNs) still are unable to capture the complex neurocomputational properties of the biological neurons. To fill in this gap, the neuromorphic community has introduced the third generation of ANNs known as SNNs. SNNs, in contrast to the ANNs, capture more closely the functionality of the nervous system by taking into account not only the spatial but also temporal aspects of the input data for the construction of the computational model. The sparse and asynchronous communication in SNNs allows data processing in a massively parallel fashion [22]. Moreover, the low power consumption, fast inference, and event-driven information processing of SNNs make them a suitable candidate for efficient implementation of deep neural networks/machine learning tasks where energy efficiency is a prime requirement. The following sections describe the experiment and methods applied in this work for the analysis of SNNs.

\section{A. Neural Engineering Object (Nengo) Simulator}

Neural Engineering Object (Nengo) is a neural network tool for simulating large-scale neural systems. It has shown applications in cognitive science, psychology, Artificial Intelligence and neuroscience [23]. For simulating deep neural networks Nengo offers NengoDL [24] that allow simple merging of TensorFlow library, hence, providing usage to rich features such as convolutional layers. Nengo is based 
on a neural engineering framework (NEF) to develop spiking neuron models with enormous applications in machine learning and deep learning such as, image classification [25], action selection [26], inductive reasoning [26], speech production [27], motor control [28], and planning with problem solving [29].

\section{B. Proposed SNN Architecture}

The proposed SNN architecture is shown in Fig. 3. Since our main objective is to make the model resource-efficient in terms of computation and energy, our model uses LIF neurons. To construct the proposed model, we use NengoDL [24] which offers a differential approximation of the firing rate of the LIF neurons, allowing the training to be done in a conventional backpropagation manner. We use SoftLIF [30] activation (an approximation to LIF) with a multi-class cross-entropy function as an objective function.

The first layer of the SNN mimics the discrete Fourier transform (DFT). Since the DFT is a linear transformation and can be represented using two successive multiplications, each DFT dimension can be represented by a single dense layer. Let the input data have $N_{s} \times N_{f}$ dimensions, where $N_{s}$ is the total number of samples per chirp and $N_{f}$ is the total number of frames. Since the DFT is complex, the layer has $2 N_{s} \times N_{f}$ nodes to compute both the real and imaginary values. The weights of the layer are calculated using the DFT trigonometric equation:

$$
D_{k}=\sum_{l=0}^{L-1} X_{l}\left[\cos \left(\frac{2 \pi}{L} k l\right)-i \sin \left(\frac{2 \pi}{L} k l\right)\right],
$$

where $k$ and $l$ take values between 0 and $N_{S}-1$. In matrix form equation (4) can be written as:

$$
\boldsymbol{D}=\left(\boldsymbol{W}_{\boldsymbol{R}}+i \boldsymbol{W}_{\boldsymbol{I}}\right) \boldsymbol{X}
$$

where $\boldsymbol{D}$ is the result of the transform, $\boldsymbol{X}$ is the input vector, and $\boldsymbol{W}_{\boldsymbol{R}}$ and $\boldsymbol{W}_{\boldsymbol{I}}$ are the real and imaginary coefficients.

The output of the first layer is then fed to convolutional layer of filter size 3 and a total number of 16 filters with stride 2. This is followed by LIF as a non-linearity function, which converts the output into spikes. Next is a fully connected layer with 16 neurons appended with LIF as an activation function. Finally, we have a classification layer with 4 neurons as the model output.

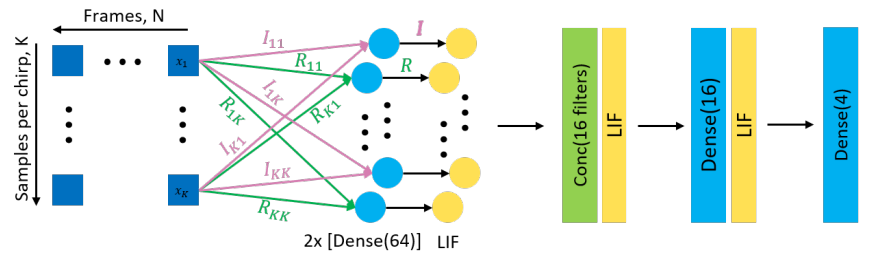

Fig. 3: The proposed spiking neural network architecture.

The softLIF activation is replaced with LIF to make the network a spiking one during the testing phase. The weights and biases are extracted from the trained model and used in connections of LIF neurons. In order to obtain an accurate measure of the spiking neurons over time, the test inputs are presented multiple times/steps to the network.

\section{EXPERIMENTAL RESULTS AND DISCUSSION}

\section{A. Dataset}

We used the dataset from [17], [18] for our experiments. The dataset contains 7819 swipes of 4 different hand gestures: 1) downup (moving hand in up direction) 2) top-down (moving hand in down direction) 3) left-right (moving the hand towards right) and 4) rubbing two fingers, as shown in Fig. 4. The dataset is divided into $80 \%$ training dataset and $20 \%$ testing dataset respectively.

\section{B. Results}

The performance of the proposed system is evaluated using classification accuracy. The system achieves a similar average accuracy of $98.1 \%$ as is achieved by the state-of-the-art models [17], [18] over random trials as shown in Table 2. Furthermore, it can be seen that our proposed architecture has significantly low latency as compared to the state-of-the-art [17], [18].

Table 2: Classification accuracy of the proposed SNN vs. other SNN models

\begin{tabular}{ccccc}
\hline Approaches & Input type & Input Size & Accuracy & Latency (ms) \\
\hline SNN [17] & Range over time image & $64 \times 32$ & $98.5 \%$ & 212.5 \\
SNN [18] & Range over time image & $64 \times 32$ & $97.5 \%$ & 189 \\
Proposed SNN & ADC & $64 \times 32$ & $98.1 \%$ & 12 \\
\hline
\end{tabular}

\section{Discussion}

This letter presents an SNN-based gesture sensing system. In contrast to conventional deepNets, SNNs not only focus on the static values but also on the occurrence times of these static values [31]. SNNs are energy efficient because they use fewer neurons as compared to artificial neural networks. Moreover, SNNs are fast, scalable and hardware-friendly and hence, very cost-effective. Compared to previous SNNs solutions, the proposed solution only uses the raw data of the target and avoids the necessity of the slow-time and fast-time FFT operations. Moreover, in contrast to previous approaches, which use range over time images of $64 \times 32$ dimensions with 32 chirps per frame, the proposed system relies only on a single chirp per frame which reduces the computation cost with a similar level of accuracy for classifying 4 gestures as shown in Table 2. This performance of the proposed system is attributed to the use of spatio-temporal information encoding by SNN, exploiting the network dynamics for learning. Moreover, the end-to-end latency of our proposed SNN is $17.7 \times$ faster than the state of the art methods. Figure 4 shows the examples of 4 different gesture samples and their firing choice by the model. It can be seen that after a few time steps the SNN starts firing for a sample from the correct classes. This is because presenting each image for longer allows integrating spikes over a longer time period and thus achieves better accuracy.

To determine its energy efficiency, we examine the energy consumption per classification. To achieve this, we used the hardware metrics of the $\mu$ Brain chip defined in [32] and mathematically given as:

$$
E_{c}=N_{\text {spikes }} \times E_{\text {spikes }}+\delta T \times P_{\text {leakage }}
$$

where $E_{c}$ is the energy consumed per classification, $N_{\text {spikes }}$ is the maximum number of spikes during classification, $E_{\text {spikes }}=2.1 \mathrm{pJ}$ is the energy per spike, $P_{\text {leakage }}=73 \mu \mathrm{W}$ is the static leakage power 


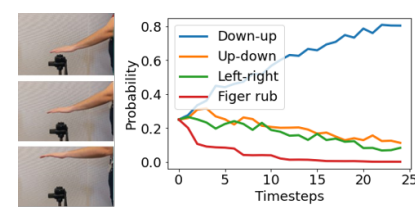

(a)

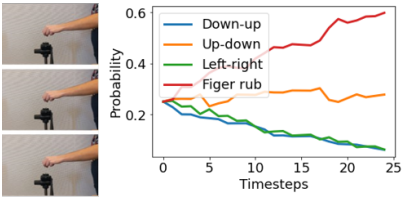

(c)
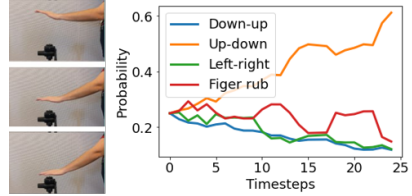

(b)
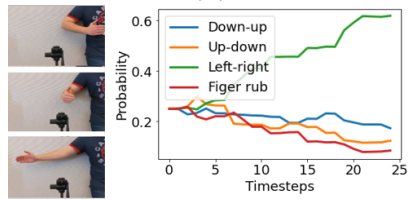

(d)
Fig. 4: Examples of 4 different gestures, (a) down-up, (b) up-down, (c) finger rub, (d) left-right along with the SNN model class firing probability over time.

and $\delta T$ is the inference time. Assuming the $\delta T$ to be $28 \mathrm{~ms}$, the energy consumption per classification of the proposed system is $E_{c}=2.05 \mu \mathrm{J}$.

\section{CONCLUSION}

We present a novel SNN-based gesture sensing system using $60 \mathrm{GHz}$ frequency modulated continuous wave radar. In contrast to state-of-the-art methods that operate on range over time images, the proposed method only works with raw ADC data thus avoiding the overhead of slow-time and fast-time FFTs, which makes the model $17.7 \times$ faster in end-to-end processing. The evaluation of our proposed SNN architecture on 4 gestures has a similar level of accuracy performance in comparison to the state-of-the-art, thus making the proposed system favorable for low latency and low power embedded implementations. In the future, we aim to implement SNNs for more and complex gestures for real-time inference.

\section{ACKNOWLEDGMENT}

This work has received funding from the Electronic Components and Systems for European Leadership Joint Undertaking under grant agreement No 826655 (Tempo) and by the German Federal Ministry of Education and Reseach (BMBF) within the KI-ASIC project (16ES0992K).

\section{REFERENCES}

[1] P. Molchanov et al., "Multi-sensor system for driver's hand-gesture recognition," in 11th IEEE FG, vol. 1, 2015, pp. 1-8.

[2] A. Malima et al., "A fast algorithm for vision-based hand gesture recognition for robot control," in IEEE 14th SIU, 05 2006, pp. 1-4.

[3] D.-S. Tran et al., "Real-time hand gesture spotting and recognition using RGB-D camera and 3D convolutional neural network," Applied Sciences, vol. 10, p. 722, 012020.

[4] V. Lammert et al., "A $122 \mathrm{ghz}$ ism-band fmcw radar transceiver," in 2020 German Microwave Conference (GeMiC), 2020, pp. 96-99.

[5] V. Issakov et al., "A highly integrated d-band multi-channel transceiver chip for radar applications," in IEEE BCICTS, 2019.

[6] J. Rimmelspacher et al., "Low power low phase noise $60 \mathrm{GHz}$ multichannel transceiver in $28 \mathrm{~nm}$ CMOS for radar applications," in IEEE RFIC, 2020, pp. 19-22.

[7] M. Pavlo et al., "Online detection and classification of dynamic hand gestures with recurrent 3d convolutional neural networks," in IEEE CVPR, 2016.

[8] S. Rautaray et al., "Vision based hand gesture recognition for human computer interaction: a survey," Artificial Intelligence Review, 2015.

[9] A. Santra and S. Hazra, Deep Learning Applications of Short Range Radars. Artech House, 2020.
[10] Z. Zhang et al., "Latern: Dynamic continuous hand gesture recognition using fmcw radar sensor," IEEE Sensors Journal, 2018.

[11] M. Arsalan et al., "Character recognition in air-writing based on network of radars for human-machine interface," IEEE Sensors Journal, 062019.

[12] M. Arsalan, A. Santra et al., "Air-writing with sparse network of radars using spatio-temporal learning," in 25th ICPR, 2021, pp. 8877-8884.

[13] M. Arsalan et al., "Radar trajectory-based air-writing recognition using temporal convolutional network," in IEEE ICMLA, 2020, pp. 1454-59.

[14] V. Sze et al., "Efficient processing of deep neural networks: A tutorial and survey," Proceedings of the IEEE, pp. 2295-2329, 2017.

[15] E. Hayashi et al., "Radarnet: Efficient gesture recognition technique utilizing a miniature radar sensor," in Proceedings of the CHI, 2021.

[16] M. Scherer et al., "TinyRadarNN: Combining Spatial and Temporal Convolutional Neural Networks for Embedded Gesture Recognition With Short Range Radars," IEEE IoT-J, vol. 8, no. 13, pp. 10336-46, 2021.

[17] M. Arsalan et al., "Resource efficient gesture sensing based on fmcw radar using spiking neural networks," in 2021 IEEE MTT-S IMS, 2021

[18] M. Arsalan, et al., "Radar-based gesture recognition system using spiking neural network," in 2021 26th IEEE ETFA, 2021.

[19] A. Safa, A. Bourdoux, I. Ocket, F. Catthoor, and G. G. E. Gielen, "On the use of spiking neural networks for ultralow-power radar gesture recognition," IEEE Microwave and Wireless Components Letters, pp. 1-4, 2021.

[20] $60 \mathrm{GHz}$-Infineon Technologies. [Online]. Available: https://www.infineon.com/ $\mathrm{cms} / \mathrm{en} /$ product/promopages/60GHz/

[21] S. Trotta et al., "2.3 soli: A tiny device for a new human machine interface," in 2021 IEEE ISSCC, vol. 64, Feb. 2021, pp. 42-44.

[22] N. Kasabov et al., "Dynamic evolving spiking neural networks for on-line spatioand spectro-temporal pattern recognition," Neural Networks, 2013, special Issue on Autonomous Learning.

[23] T. Bekolay et al., "Nengo: a python tool for building large-scale functional brain models," Frontiers in neuroinformatics, vol. 7, p. 48, 2014

[24] NengoDL. [Online]. Available: https://www.nengo.ai/nengo-dl/

[25] J. A. K. Ranjan, T. Sigamani, and J. Barnabas, "A novel and efficient classifier using spiking neural network," The Journal of Supercomputing, pp. 1-16, 2019.

[26] V. Senft, T. C. Stewart, T. Bekolay, C. Eliasmith, and B. J. Kröger, "Reduction of dopamine in basal ganglia and its effects on syllable sequencing in speech: a computer simulation study," Basal Ganglia, vol. 6, no. 1, pp. 7-17, 2016.

[27] B. J. Kröger, T. Bekolay, and C. Eliasmith, "Modeling speech production using the neural engineering framework," in 5 th Conference on Cognitive Infocommunications (CogInfoCom). IEEE, 2014, pp. 203-208.

[28] K. E. Friedl, A. R. Voelker, A. Peer, and C. Eliasmith, "Human-inspired neurorobotic system for classifying surface textures by touch," IEEE Robotics and Automation Letters, vol. 1, no. 1, pp. 516-523, 2016.

[29] J. Knight, A. R. Voelker, A. Mundy, C. Eliasmith, and S. Furber, "Efficient spinnaker simulation of a heteroassociative memory using the neural engineering framework," in IJCNN. IEEE, 2016, pp. 5210-5217.

[30] E. Hunsberger and C. Eliasmith, "Training spiking deep networks for neuromorphic hardware," arXiv preprint arXiv:1611.05141, 2016.

[31] N. Kasabov, Time-Space, Spiking Neural Networks and Brain-Inspired Artificial Intelligence. Springer Berlin Heidelberg, 2018.

[32] J. Stuijt et al., " $\mu$ brain: An event-driven and fully synthesizable architecture for spiking neural networks," Front. Neurosci., vol. 15, p. 538, May 2021. 